\title{
Advanced Active Power Conditioning to Improve Power Quality in Microgrids
}

\author{
${ }^{1}$ Rameez Khan \\ ${ }^{1}$ PG Students, \\ Department of Electrical Engineering, \\ Veermata Jijabai Technological Institute, \\ Mumbai
}

\begin{abstract}
In the recent time, Wind energy conversion systems have become an important topic for the research in the field of renewable energy sources with microgrid. Wind power generation has a major challenge to focus on which is power quality problem and at the same time its connection with the distribution network. The converter and rectifier which is used in the microgrid are to convert $\mathrm{AC}$ to $\mathrm{DC}$ to $\mathrm{AC}$ for the interconnection of load and wind power to microgrid and for producing the significant harmonics. In power network, the quality of the power becomes poor due to the impact of harmonics in current and voltage. If a microgrid is a weak electrical grid than it may easily lead to the disturbances which need to be taken care of. Therefore, a three-phase Active Power Conditioner (APC) is required to enhance the power quality in microgrid which would be based on the renewable energy and APC here interact between the AC bus of a microgrid and the renewable energy sources which basically means acting as a interface. To correct the power factor, APC should use an improved control strategy by which it is possible to inject some energy in the microgrid and compensation of the current harmonics. By the improved control strategy, even if the load is unbalanced it allows the line current at the point of common coupling (PCC) to be sinusoidal and balanced. To validate the improved control strategy, we use the simulation.
\end{abstract}

Index Terms-Active Power Conditioner, Microgrids, Renewable Energy, Current control, AC-DC-AC Converter.

\section{INTRODUCTION}

Technological advances in power electronics have created opportunities for the renewable sources to be exploited in different configurations. The power electronic interface allows renewable sources to be connected with the distribution grid or interconnected with other renewable and non-renewable generators, storage systems and loads in a microgrid [1]. A microgrid is different from a main grid system which can be considered as an unlimited power so that load variations do not affect the stability of the system. On the contrary, in a microgrid, large and sudden changes in the load may result in voltage transient of large magnitudes in the AC bus. Moreover, the proliferation of switching power converters and nonlinear loads with large rated power can increase the contamination level in voltages and currents waveforms in a microgrid, forcing to improve the compensation characteristics required to satisfy more stringent harmonics standards.

A possible solution to overcome the above-mentioned drawback is to use the APC as a power interface between the renewable energy sources and the AC bus of the microgrids as shown in Fig. 1.

The APC has proved to be an important alternative to compensate current and voltage disturbances in power distribution systems [2], [3]. Different APC topologies have been presented in the technical literature [4], but most of them are not adapted for microgrids applications.

This paper presents an APC used to improve the power quality in a microgrid. The attention will be mainly focused on the innovative control strategy, which allows injecting energy in the microgrid, compensating the current harmonics, correcting the power factor and balancing the supply voltage at the PCC. The validity of the control strategy has been proved through many simulation tests using SimPower Systems from MATLAB.

Active power Conditioners:

The huge gap between energy demand and supply has put extra ordinary stress on electrical supply infrastructure. Utilities are introducing increasingly stricter regulations on industries with specific focus on power quality.

Common measures of poor power quality are:

- High current and voltage harmonics

- Load imbalances

- Low power factor

- Poor power quality has severe cost implications for the industries:

- Frequent failures of equipment

- Reduced lifetime of equipment

- Production losses

- $\quad$ Reduced safety levels of installations

- Increased carbon footprint

- Non-compliance with utility regulations

In addition to financial losses there are costs incurred due to extra $\mathrm{kWh}$ losses in typical network components such as transformers, cables and motors. Traditionally, low power factor has been considered to be the only parameter responsible for introducing inefficiencies in the electrical systems. More emphasis was given on conventional passive solutions like APFC, Fixed Capacitor Banks etc. However, these solutions do not address the real problem of presence of high harmonics in the electrical network. 


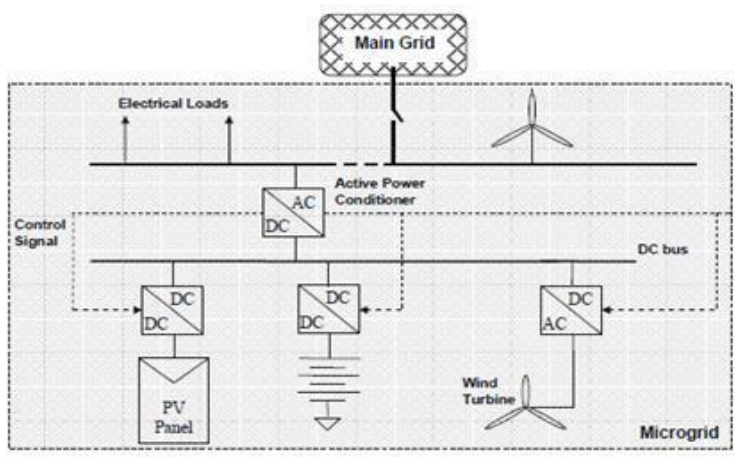

Fig. 1APC for microgrid applications Harmonics are generated due to the increasing use of nonlinear loads by the modern industries.

Some of the commonly found non-linear loads are:

- Variable frequency drives

- Welding loads

- Electric Arc Furnaces

- Computers

Typical industries affected by harmonic issues are:

- Automotive Industry

- Cement Industry

- Steel/Foundries

- $\quad$ Pulp Processing Industry

- $\quad$ Printing Industry

Power Quality relates to the amplitude, frequency and distortion of the supply system. While the amplitude and frequency of the supply is largely controlled by the utility, the distortion of the wave (voltage or current) is attributed to the user (of the power) or the loads.

- Harmonic currents increase the rms current in electrical systems and deteriorate the supply voltage quality.

- $\quad$ They stress the electrical network and can potentially damage equipments.

\section{Load Unbalance:}

Commercial complexes and industries sometimes have a large number of single phase and two phase loads which results in the unbalanced loading of transformers. Unbalance leads to the flow of negative sequence current in the network.

- Negative sequence current will cause voltage unbalance (known as negative sequence voltage). Likewise, an unbalanced 3-phase voltage will cause unbalanced current in other loads.

- Negative sequence current reduces the overall system capacity-transformers, cables, and bus capacity are lost.

Low Power Factor:

Maintaining a high-power factor is important for reducing utility bills and reducing the current loading of the safety devices and conductors within the facility. However, correction of power factor is fast becoming very difficult due to the increased use of nonlinear loads.
- Use of power factor correction capacitors in electrical systems where nonlinear loads are present can be hazardous to the capacitors and all other equipment connected in the network.

- Capacitors can lead to electrical resonance in the presence of harmonics. Resonance can cause very high peak AC voltages detrimental to all loads.

- Premature tripping of circuit breakers; nuisance faulting of equipment; or damage to equipment.

In all cases, plant interruptions occur. When electrical systems contain nonlinear loads that exceed about 50

$\%$ of the total load, the solution for poor DPF is no longer viable with PF capacitors. DPF correction must be achieved with alternate mean. One method is using active power conditioners.

\section{II.CONTROL SCHEME}

The proposed control scheme is illustrated in Fig. 2, which includes three modules: the harmonic cancellation

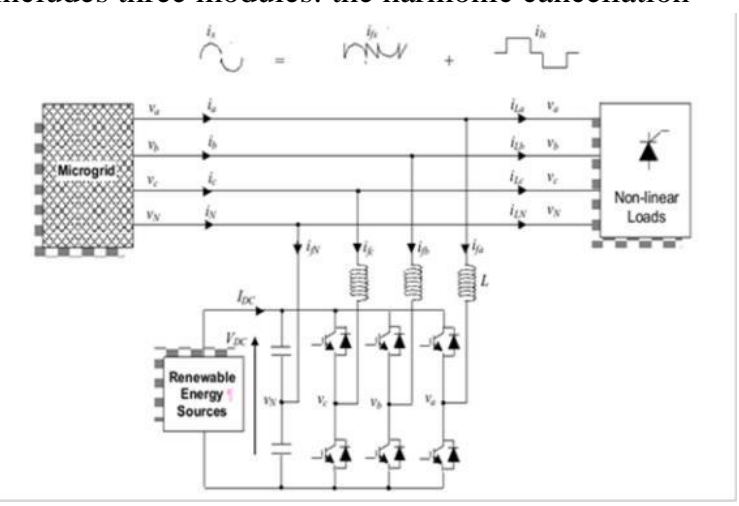

Fig-2 APC Topology

module, the reactive power compensation module, and the DC capacitor voltage control module.

The harmonic cancellation module generates harmonic compensating signal and provides the fundamental current information to the reactive power compensation module. The reactive power compensation module calculates reactive power compensating signal. The DC capacitor voltage control module regulates the voltage across the DC capacitor. All compensating signals are summed up at the abc-frame to form the reference signal for the hysteresis current controller [11]. Finally, gating signals are generated for the IGBT based switching devices. From above introduction, we can see that the harmonic cancellation and the reactive power compensation modules are used towards the nonlinear load, while the DC voltage control module is used for the active power filter. This is the reason why the signs assigned to the three signals are ,,+"e, ,,+e, and ,,-

Generally, four-wire APCs have been conceived using fourleg converters [5]. This topology has proved better controllability [6] than the classical three-leg four-wire 
converter but the latter is preferred because of its lower number of power semiconductor devices. In this paper, it is shown that using an adequate control strategy, even with a simple three-leg four-wire system, it is possible to mitigate disturbances like voltage unbalance. The topology of the investigated APC and its interconnection with the microgrid is presented in Fig. 2. It consists of a three-leg four-wire voltage source inverter. In this type of applications, the VSI operates as a current controlled voltage source. In order to provide the neutral point, two capacitors are used to split the DC-link voltage and tie the neutral point to the mid-point of the two capacitors. This topology allows the current to flow in both directions through the switches and the capacitors, causing voltage deviation between the DC capacitors. i_fa+i_fb+i_fc $=$ i_fN

where:

i_fa,i_fb,i_fc are phase APC currents and i_fN is the APC neutral current.

Therefore, the total DC voltage will oscillate not only at the switching frequency but also at the corresponding frequency of the neutral current. As shown in [2], if the current control is made by hysteresis, the above-mentioned drawback can be limited with a dynamic offset level added to both limits of the hysteresis band.

For the investigated topology presented in Fig. 2, the

current at (PCC) is:

i_x $=$ i_l $1 \mathrm{x}+\mathrm{i} \_\mathrm{f} x$

where:

i_x,i_lx,i_fxare the microgrid side current, the load current, and the APC current respectively. The $x$ index points the $a, b$ and c current phases.

The instantaneous load current is:

i_lx $=$ i_l $x^{\wedge} 1+i \_1 x k+i \_l x q$

where:

- i_lx^1the fundamental active current component;

- i_lxk the addition of current harmonics;

- i_lxq the reactive current component.

The three-phase APC current is given by:

ifx ifx + if $x$

i_f $\mathrm{x}^{\wedge} 1$-the fundamental conditioner current component;

i fx - the deforming component of the current.
As shown in Fig. 2 the current drawn from the grid has to be sinusoidal and moreover, in phase with the voltage at PCC. Consequently, the control strategy for the APC has to be designed in order to ensure a sinusoidal wave for the grid current.

i $1 x+i l x+i l x q+i f x+i f x \quad$ i $x$

The APC switches generate undesirable current harmonicsaround the switching frequency and its multiples. Consideringthe switching frequency of the APC sufficiently high, theseundesirable current harmonics can be filtered with the LRpassive filter.

\section{CONTROL OF THE APC A. Control}

\section{Strategy}

There are many ways to design a control algorithm for an APC [7][8]. Generally, the controller design is made considering that the grid voltage at the PCC is balanced. In a microgrid, the supply voltage itself can be distorted and/or unbalanced. Consequently, the controller of an APC used to improve the power quality in the microgrid has to be designed according to the weakness of this kind of grid.

The proposed control algorithm is a compensation method that makes the APC compensate the current of a non-linear load by forcing the microgrid side current to become sinusoidal and balanced (Fig. 3). The controller requires the three-phase grid current (i_a,i_b,i_c), the three-phase voltage at the PCC (v_a,v_b,v_c) and the DC-link voltage (V_DC). As shown in Fig. 3, the sinusoidal waveform and the

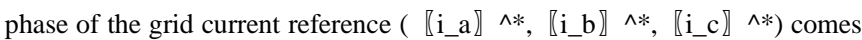
from the line voltage thanks to a PLL. The magnitude of the same current is obtained by passing the error signal between the DC-link voltage (V_DC) and a reference voltage ( $\left.\llbracket \mathrm{V} \_D C \rrbracket^{\wedge *}\right)$ through a PI controller.

\section{B. Switching control}

As shown in Fig. 3, the hysteresis control has been used to

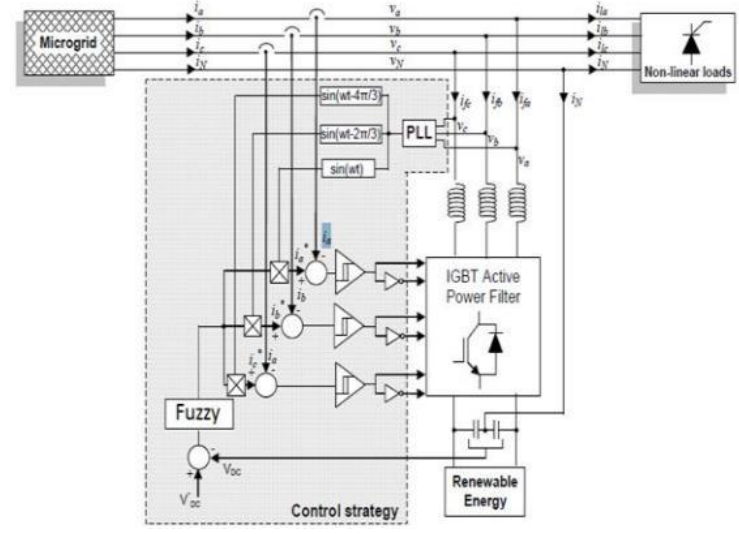

Fig-3. APC Control Strategy 
keep the controlled current inside a defined band around the references. The status of the switches is determined according to the error. When the current is increasing and the error exceeds a certain positive value, the status of the switches changes and the current begins to decrease until the error reaches a certain negative value. Then, the switches status changes again. Compared with linear controllers, the non-linear ones based on hysteresis strategies allow faster dynamic response and better robustness with respect to the variation of the non -linear load. A drawback of the hysteresis strategies is the switching frequency which is not constant and can generate a large side harmonics band around the switching frequency.

To avoid this drawback, the switching frequency can be fixed using different solutions like variable hysteresis bandwidth [9] or modulated hysteresis [10] But this is not the object of this paper.

\section{IV.RESULTS AND DISCUSSION}

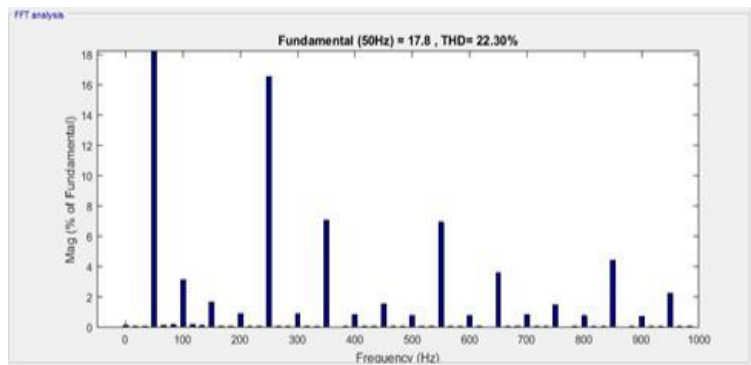

Fig. 4 i_l Fuzzy with 0.1 sstart time

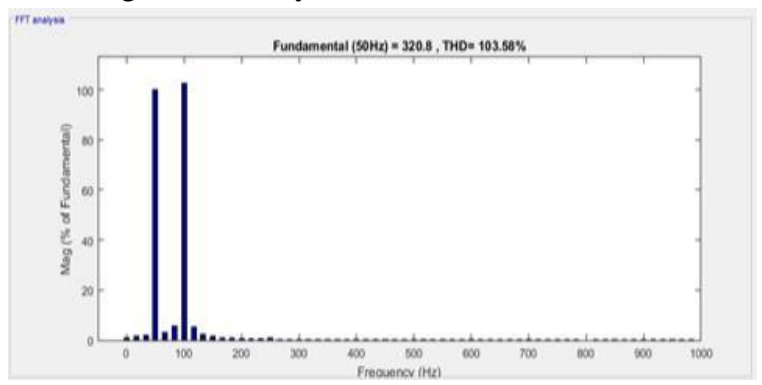

Fig. 5i_s Fuzzy with 0.1sstart time

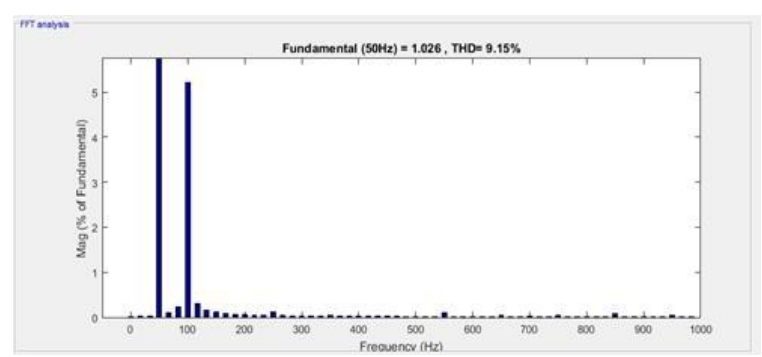

Fig. 6V_1 Fuzzy with 0.1sstart time
Fig. 7V_s Fuzzy with 0.1sstart time

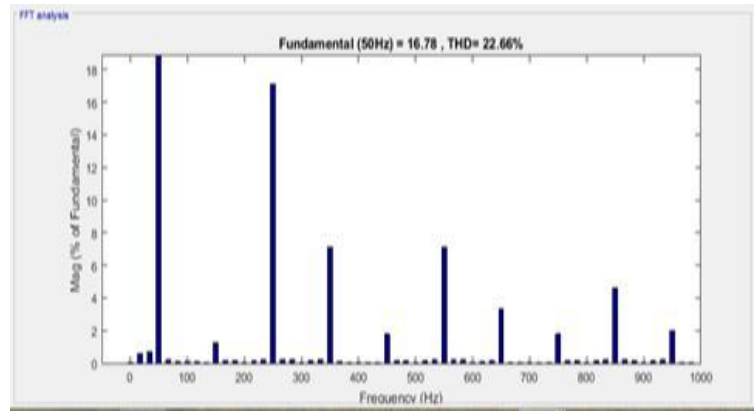

Fig. 8i_1 Fuzzy with 0.5sstart time

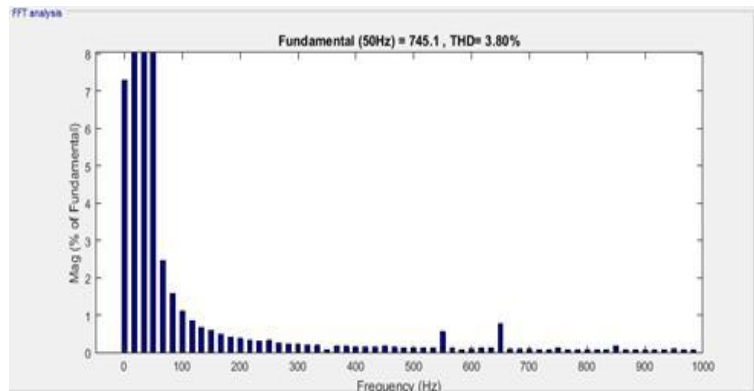

Fig. 9i_s Fuzzy with 0.5sstart time

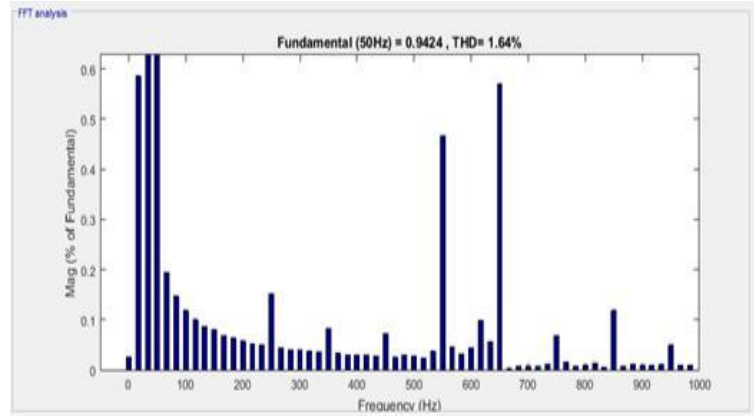

Fig. 10V_1 Fuzzy with 0.5 sstart time

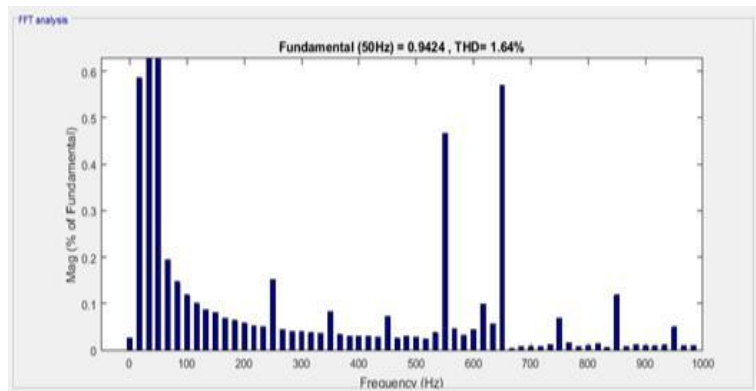

Fig. 11V_s Fuzzy with 0.5 sstart time
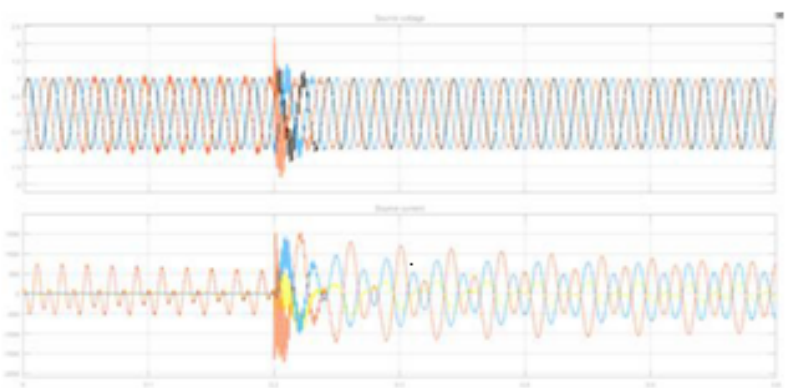

Fig. 12Current and voltage at the source 


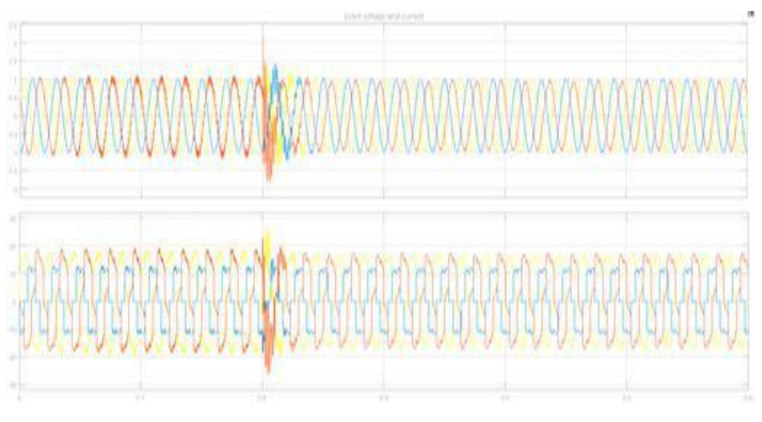

Fig. 13 Current and voltage at the load

During this case study, the APC is investigated using a threephase diode bridge rectifier with a $60 \Omega$ resistor in series with a $0.1 \mathrm{mH}$ inductor at the DC side. The power delivered by the renewable sources is $3 \mathrm{~kW}$ and the load requires 5 $\mathrm{kW}$.

\section{V.CONCLUSION}

To eliminate the harmonic current and reactive power problem, an improvised control strategy is proposed. This control schemes correct the power factor and improve the power quality by injecting some energy in the microgrid and by compensating both harmonic current reactive power in the system. An Improvised control strategy is very straightforward to understand and simple to implement if we compare it with the conventional control schemes already present. The results from the simulation prove that an improvised control strategy has good steady state and dynamical performance. In the future, the research is required to find the optimal control strategy for the cases where harmonic and reactive power compensation requirement exceeds the rating of the active filter.

\section{REFERENCES}

[1] H. Ibrahim, A. Ilinca and J. Perron, "Energy Storage Systems Characteristics and Comparisons,"ElsevierRenewable\& SustainableEnergy Reviews, Vol. 12, pp. 1221-1250, 2008.

[2] M. Abdusalama, P. Poureb, S. Karimia and S. Saadatea "New digital reference current generation for shunt active power filter under distorted voltage conditions," Electric Power Systems Research, vol. 79, pp 759-765, May 2009.

[3] M.Montero, E.R. Cadaval, F. Gonzalez, "Comparison of control strategies for shunt active power filters in three-phase four-wire systems ", IEEE Transactions on Power Electronics, vol. 22, pp. 229236, January 2007.

[4] B. Singh, K. Al-Haddad, A. Chandra "A Review of Active Filters for Power Quality Improvement" IEEE Transactions on Power Electronics,vol. 46, pp. 960-971, October 1999.

[5] J. Chen, F. Liu , S. Mei, "Nonlinear disturbance attenuation control for four-leg active power filter based on voltage source inverter," Journal ofControl Theory and Applications vol. 3 pp. 261-266, August 2006.

[6] M. Ucara and E. Ozdemir "Control of a 3-phase 4-leg active power filter under non-ideal mains voltagecondition,"ElectricPower Systems Research, vol. 78, pp. 58-73, January 2008.

[7] A. Zhikang, S. Wenji Z. Ruixiang and F. Chunming Tu "Development of Hybrid Active Power Filter Based on the Adaptive Fuzzy Dividing Frequency-Control Method," IEEE Transactions on Power Delivery, vol.24, pp. 424 - 432, January 2009. 